\title{
EVALUATION OF GLOVE INTEGRITY AFTER MULTIPLE WASHES WITH DISINFECTANT
}

W.L. Chai, Z.Y.M. Yusof, J. Marhazlinda, C.N. Shim, M.Y. Lee, P. Nambiar. Evaluation of Glove Integrity after Multiple Washes with Disinfectant. Annal Dent Univ Malaya 2012; 19(2): 51-55.

\section{ABSTRACT}

Background: In an academic setting due to financial constrain, it is not uncommon during non-surgical procedures dental students and clinical supervisors wash their gloved hands with disinfectants in between patients or when touching on non-contaminated objects. Whether this practice could cause any deterioration of the glove and expose clinicians and patients to infectious micro-organisms was a concern. Aim: The aim of this study was to investigate the effect of multiple washes of gloved hands with a disinfectant on the integrity of the gloves. Methods: Three brands of commonly used gloves in a dental school were tested for leaks after multiple washes with a disinfectant. Thirty pairs of each type of gloves were subjected to $0,1,5,10,20$ and 30 washes with a disinfectant solution at a 5-minute interval between each wash. After each washing cycle, the gloves were filled with 1L of water and hanged for 2 minutes to observe any signs of water leaks. Results: The results showed that the type of gloves and number of washes were significantly associated with the leakage rates $(p<0.001)$. Washing of gloves for more than 5 times were at least 6 times higher to suffer from leakage $(\mathrm{OR}=6.23,95 \% \mathrm{CI}=2.14-18.08)$. Powdered gloves were almost 13 times higher to leak in all washes $(\mathrm{OR}=12.78,95 \% \mathrm{CI}=4.40-37.14)$ and were almost 25 times more likely to leak when washed for more than 5 times $(\mathrm{OR}=24.92,95 \% \mathrm{CI}=5.79-107.21)$ when compared to the non-powdered gloves. Conclusion: The practice of washing gloved hands with a disinfectant deteriorates the integrity of the gloves.

Key words: Cross infection, disinfectant, glove, leakage, micropores

\section{INTRODUCTION}

The use of examination gloves in clinical practice has risen dramatically in response to an increased need to protect health care workers and patients from the possibility of transmission of various infectious diseases through contact with body fluids and also against hazardous chemicals (1-6). Various materials have been used in manufacturing gloves such as natural rubber latex or synthetic materials like nitrile, polyvinyl chloride or polyurethane (7). Some

\section{Original Article}

\begin{tabular}{|c|}
\hline $\begin{array}{l}\text { W.L. Chai }{ }^{1} \text {, Z.Y.M. Yusof }{ }^{2} \text {, J. Marhazlinda }{ }^{2} \text {, } \\
\text { C.N. Shim }{ }^{3} \text {, M.Y. Lee }{ }^{3} \text { and P. Nambiar }\end{array}$ \\
\hline $\begin{array}{l}{ }^{1} \text { Department of General Dental Practice, } \\
\text { Faculty of Dentistry, University of Malaya, } \\
\text { 50603, Kuala Lumpur, Malaysia. } \\
\text { E-mail: chaiwl@um.edu.my } \\
\text { Tel: 603-7967 4548 } \\
\text { Fax:603-7967 } 4575\end{array}$ \\
\hline $\begin{array}{l}{ }^{2} \text { Department of Community Dentistry, } \\
\text { Faculty of Dentistry, University of Malaya, } \\
\text { 50603, Kuala Lumpur, Malaysia. }\end{array}$ \\
\hline${ }^{3}$ Ministry of Health, Malaysia \\
\hline Corresponding author: Dr. Chai Wen Lin \\
\hline
\end{tabular}

chemicals that come in contact with the gloves may react with the glove material, causing damage to the glove surface, creating micropores which may lead to penetration of harmful bacteria or viruses to the skin, and subjected the operators or patients to contamination $(1-6,8)$.

It is a standard infection control policy that the clinical gloves should be disposed after use (9). However, in some teaching institutions due to costing factors, during non-bloody procedures, the students and staff would wash their gloved hands with a disinfectant, rather than dispose it every time when touching on non-contaminated surfaces such as pen, patient's record, working bench and drawers. Thus, a single pair of gloved hands could have been washed several times during the non-surgical procedures. Although hand wash disinfectant is a relatively mild and non-corrosive agent, a study had detected that the ethanol component from the hand disinfectant permeated through latex, vinyl, nitrile and synthetic elastomer after 10 mins of exposure (2). The aim of this study was to evaluate the effect of multiple washes of gloved hands with a hand disinfectant on the integrity of 3 types of non-sterile latex gloves, and to determine the number of washes that result in a positive leak of gloves.

\section{MATERIALS \& METHODS}

A modified water-leak test based on Food and Drug Administration (FDA) protocol was used to determine the leakage of non-sterile latex gloves after few washes with disinfectant (10). The FDA recommends two-part 
testing protocol where the gloves are first inspected visually and then subjected to a water-leak test. Three brands of non-sterile latex examination gloves namely Vandaier Latex ${ }^{\circledR}$ (VL) (powdered, smooth), I-Vision Latex $^{\circledR}$ (IV) (non-powdered, rough) and Cross Protection Latex ${ }^{\circledR}$ (CP) (non-powdered, rough) were tested. All gloves were manufactured in Malaysia. Before the experiment, the gloves were visually inspected and tested with air leaking test by inflating the gloves with air from the dental chair to a size of about a 5-cm diameter, and then felt for any air leakage. Any defective gloves were discarded. Five volunteers were asked to remove all rings and cut their fingernails short, polished and smoothened. All hands were washed and dried with a clean towel before wearing the gloves. The volunteer wore a pair of the gloves and washed their hands with $2 \mathrm{ml}$ of disinfectant solution (ESEMTAN $^{\circledR}$ Wash Care, Schulke \& Mayr Pte Ltd, Asia) and rinsed under running water in a standard manner. Thirty pairs of gloves were used in each washing cycle, i.e. $0,1,5,10,20$, and 30 times, with a 5 -min interval in each wash, resulting in a total of 180 pairs of gloves were used for each brand of gloves.

After the required number of washes, the gloves were removed inside-out and tested for water leakage. The gloves were filled carefully with $1 \mathrm{~L}$ of water. After 2 minutes, water absorbent paper was used to wipe on the glove surfaces to detect any signs of water leaks.

Data was analysed using SPSS version 12.0. Descriptive analysis was performed to provide general frequencies and percentage of gloves showing sign of water leakage. Gloves were categorized into nonpowdered (IV and CP) and powdered (VL), while the number of washes was categorized into five times or less $(\leq 5)$ and more than five times $(>5)$. Chi square or Fisher exact test was used to compare leakage rates between the different type of gloves and the different number of washes. Odd ratios (OR) were used to assess the effect of both variables on the integrity of the gloves. Finally, stratified analysis was carried out to determine the association between the type of gloves and glove leakage percentage when the number of washes was controlled. Level of statistical significance was set at 0.05 .

\section{RESULTS}

Following initial screening of the gloves with airleaking test, the IV latex gloves showed no sign of air leaking, while $0.5 \%$ of VL and $3.0 \%$ of CP latex gloves were rejected due to presence of air-leaking. The finding from the water-leaking test after multiple washes was presented in Table 1 . There were $6.7 \%$ of VL $(n=24), 1.1 \%$ of $\mathrm{CP}(\mathrm{n}=4)$ and none in IV group showed sign of water leakage through the gloves. Most water leakage happened after 20 washes (Table 1) and occurred on the thumb and index fingertips region of the gloves.

Table 2 shows the associations between types of gloves, number of washes and water leakage rates. In total, 24 leaks were observed in powdered gloves compared to 4 leaks in non-powdered gloves whose difference was statistically significant $(\mathrm{p}<0.001)$ (Table 2-a). The odds ratio (OR) indicates the odds of having leaked gloves were almost 13 times greater for powdered gloves $(\mathrm{OR}=12.78,95 \% \mathrm{CI}=4.40-37.14)$. In other words, powdered gloves were almost 13 times more likely to leak when washed with a disinfectant compared to non-powdered gloves. Regardless of the types of gloves, leakage rates were also found to be significantly higher when the gloves were subjected to more than 5 washes with disinfectant $(\mathrm{p}<0.001)$ (Table 2-b). The OR of having leaked gloves were at least 6 times greater when the gloves were subjected to more than 5 washes $(\mathrm{OR}=6.23,95 \% \mathrm{CI}: 2.14-$ 18.08). Between the 2 types of gloves, frequent washes for more than 5 times led to significantly higher leaks in powdered gloves compared to non-powdered gloves $(\mathrm{p}<0.001)$ with the risk of leakage increased for almost 25 times more for powdered gloves (OR $=24.92,95 \%$ $\mathrm{CI}=5.79-107.27$ ) (Table 2-b-i). However, no significant

Table 1. Percentage of three non-sterile latex gloves showing sign of water leakage after a number of hand washes

\begin{tabular}{|c|c|c|c|c|c|c|}
\hline \multirow{4}{*}{$\begin{array}{l}\text { Number of } \\
\text { washes }\end{array}$} & \multicolumn{6}{|c|}{ Water leakage } \\
\hline & \multirow{2}{*}{\multicolumn{2}{|c|}{$\begin{array}{c}\text { Powdered latex } \\
\text { Vandier (VL) }\end{array}$}} & \multicolumn{4}{|c|}{ Non-powder latex } \\
\hline & & & \multicolumn{2}{|c|}{ I- Vision (IV) } & \multicolumn{2}{|c|}{$\begin{array}{c}\text { Cross Protectior } \\
\text { (CP) }\end{array}$} \\
\hline & number & $\%$ & number & $\%$ & number & $\%$ \\
\hline 0 & 0 & 0 & 0 & 0 & 2 & 3.4 \\
\hline 1 & 2 & 3.4 & 0 & 0 & 0 & 0 \\
\hline 5 & 0 & 0 & 0 & 0 & 0 & 0 \\
\hline 10 & 2 & 3.4 & 0 & 0 & 0 & 0 \\
\hline 20 & 12 & 20.0 & 0 & 0 & 2 & 3.4 \\
\hline 30 & 8 & 13.0 & 0 & 0 & 0 & 0 \\
\hline TOTAL & 24 & 6.7 & 0 & 0 & 4 & 1.1 \\
\hline
\end{tabular}


Table 2. Associations between types of gloves, number of hand washes and glove leakage rates

\begin{tabular}{|c|c|c|c|c|}
\hline \multirow{2}{*}{ Vairables } & \multicolumn{2}{|c|}{ Glove Leakage } & \multirow{2}{*}{ OR $(95 \% \mathrm{Cl})$} & \multirow{2}{*}{$p$-value ${ }^{a}$} \\
\hline & Yes Freq (\%) & No Freq (\%) & & \\
\hline \multicolumn{5}{|l|}{ (a) Types of Gloves } \\
\hline Powdered (VL) & $24(6.7)$ & 336 (93.3) & $12.78(4.40-37.14)$ & $<0.001$ \\
\hline Non Powdered (IV,CP) & $4(0.6)$ & $716(99.4)$ & & \\
\hline \multicolumn{5}{|l|}{ (b) Frequency of washes } \\
\hline More than 5 times & $24(4.4)$ & $516(95.6)$ & $6.23(2.14-18.08)$ & $<0.001$ \\
\hline 5 times and less & $4(0.7)$ & $536(99.3)$ & & \\
\hline [i] More than 5 times & & & & \\
\hline Powdered (VL) & $22(12.2)$ & $158(87.8)$ & $24.92(5.79-107.27)$ & $<0.001$ \\
\hline Non Powdered (IV,CP) & $2(0.6)$ & $358(99.4)$ & & \\
\hline [ii] 5 times and less & & & & \\
\hline Powdered (VL) & $2(1.1)$ & $178(98.9)$ & $2.01(0.28-14.39)$ & $0.604^{b}$ \\
\hline Non Powdered (IV,CP) & $2(0.6)$ & 358 (99.4) & & \\
\hline
\end{tabular}

Level of significance was set at 0.05

a Chi square test was used

${ }^{b}$ Fisher exact test was used

difference in leakage rates was observed between the two types of gloves when subjected to 5 washes or less (Table 2-b-ii).

\section{DISCUSSION}

This study was carried out to investigate the effect of multiple washes of gloved hands with a disinfectant solution on the integrity of the gloves, and to determine the number of washes which result in positive water leaks. Various methods to test the integrity of gloves such as by examining the glove surfaces under light or scanning electron microscopes, penetration tests using air inflation, water leaking, fluorescence and electric current detection, and permeability test using bacteria, viruses or dental materials have been reported (2). However instead of the gloves worn on hands, they were tested directly from the package and bypassed the practical aspect of its use. It has been demonstrated that the barrier quality of gloves is often accentuated by the application of stress, simulated use or actual clinical use (11). In a recent study, a robotic hand, which simulates the normal hand motions, had been documented (12). The use of robotic hand has the advantage of investigating the effect of highly causative chemical agents on the integrity of gloves when worn on hands. This will also reduce the risk of exposing human subjects to the causative chemical agents during the test.

In order to emulate a clinical practice in which clinicians washed gloved hands with disinfectant in between non-surgical procedures (eg. restorative works, radiograph taking), the volunteers in this study washed their gloved hands at 5-min intervals up to 30 times. The gloves were then evaluated for its integrity after the multiple washes based on the water-leak test.
The findings in this study shown that multiple washes of gloved hands with a disinfectant (ESEMTAN ${ }^{\circledR}$ Wash Care) affecting the integrity of the gloves, as shown in the water-leaking test in the VL and CP latex gloves. The disinfectant solution used in this study is a common disinfectant used in dentistry for hand washing. According to the manufacturer's manual, it contains active agents such as sodium chloride, sodium hydroxide, citric acid, lactic acid, undeclenic acid, lauric acid and phenoxyethanol. The actual chemical agent in the disinfectant solution that causes damage on the surface of gloves was not identified. In dentistry, the gloves also often in contact with other chemical agents such as acrylate monomers (4), alcohol (2), cresophene and sodium hypochlorite (13), which were reported to have damaging effect causing permeability on the gloves.

The quality of glove appeared to be closely related to the manufacturer's production quality, as this was evident when $0.5 \%$ and $3 \%$ of VL and CP gloves were rejected respectively before the experiment due to defect detected through the air test. In contrast, none was rejected when the 180 pairs of gloves were selected from the IV gloves for the experiment. In this study, the powdered smooth VL gloves suffered the highest rates of perforations (7\%) after multiple washes compared to the non-powdered rough latex gloves (CP and IV). One possible explanation to this finding was the presence of the powder in the latex gloves might damage the integrity of the gloves during the washing actions.

It was noticed in this study that the thumb and finger index areas of the gloves were the most frequent leakage areas. This could be due to the standard hand wash procedure during the experiment, in which the physical rubbing movement in these areas might damage the integrity of the glove. It is also worthy to 
note that there are other procedures in clinical situations such as holding dental instruments, stretching of the gloves while retracting the mouth or coming in contact with dental materials that could have resulted in 'greater stressed' gloves, which may subsequently amplify the deleterious effect on the gloves when washed with disinfectant.

The results of the study underline the need to evaluate infection control inclination among students and clinical supervisors in terms of glove usage. This finding indicates that washings of gloved hands with a disinfectant run a risk of damaging the protective barrier of the gloves, which subsequently exposes clinicians to chemical agents and infectious pathogens. Hence, the practice of 'recycling' of gloves by multiple washing of the gloved hand should be avoided. However, due to financial constraints, the 'reuse' of gloves was practiced in some developing countries, despite evidences against that practice (14). A study reported that the use of autoclaved reused gloves had a defect of $13 \%$ and a perforation rate as high as $82 \%$ (14).

Some clinicians, who succumb to washing their gloved hands, wear double or triple gloves, glove liners for better protection. However, this does not necessarily guarantee safety against cross infection, as reported in a systematic review that although the additional barriers significantly decreased the perforations rate to the innermost gloves, the evidence on the reduction of infection rates in patients was not clear (15).

In clinical practice, the cross infection protocol should always be strictly followed. Gloves should be removed before touching on non-contaminated surfaces such as pen, clinical models or records and then a new pair of gloves should be used for the subsequent procedures. However, if this ideal protocol could not be implemented, clinicians should at least consider changing to a new pair of gloves when a more contaminated procedure such as scaling or extraction is going to be carried out, rather than wearing the same gloves which had been washed multiple times. It is advised that changing gloves should be practiced when carried out long clinical procedures (16). In essence, the clinicians should be aware that they are not fully protected by wearing gloves especially after multiple washes of the gloved hands.

\section{CONCLUSION}

Within the limitations of the study, multiple washes of gloved hands with a disinfectant solution for more than 5 times lead to deterioration of the integrity of the gloves, which caused water leakage in the latex gloves in the powdered latex Vandier ${ }^{\circledR}$ and nonpowdered latex I-Vision ${ }^{\circledR}$ groups.

\section{ACKNOWLEDGEMENT}

This study was supported by PJP F0311/2007B grant, Institute of Research Management and Consultancy, University of Malaya, 50603, Kuala Lumpur. The authors would like to thank all the volunteers who had assisted in this study.

Note: The authors do not have financial links to any of the glove or disinfectant companies.

\section{REFERENCES}

1. Andreasson H, Boman A, Johnsson S, et al. On permeability of methyl methacrylate, 2hydroxyethyl methacrylate and triethyleneglycol dimethacrylate through protective gloves in dentistry. Eur J Oral Sci. 2003; 111(6): 529-35.

2. Baumann MA, Rath B, Fischer JH, et al. The permeability of dental procedure and examination gloves by an alcohol based disinfectant. Dent Mater. 2000; 16(2): 139-44.

3. Jordan SL, Stowers MF, Trawick EG, et al. Glutaraldehyde permeation: choosing the proper glove. Am J Infect Control. 1996; 24(2): 67-9.

4. Lonnroth EC, Wellendorf $\mathrm{H}$ and Ruyter E. Permeability of different types of medical protective gloves to acrylic monomers. Eur J Oral Sci. 2003; 111(5): 440-6.

5. Makela EA, Vainiotalo $\mathrm{S}$ and Peltonen K. The permeability of surgical gloves to seven chemicals commonly used in hospitals. Ann Occup Hyg. 2003; 47(4): 313-23.

6. Mellstrom GA, Wrangsjo K, Wahlberg JE, et al. The value and limitations of protective gloves in medical health service: part II. Dermatol Nurs. 1996; 8(4): 287-95.

7. Connor TH. Permeability of nitrile rubber, latex, polyurethane, and neoprene gloves to 18 antineoplastic drugs. Am J Health Syst Pharm. 1999; 56(23): 2450-3.

8. O'Connell KP, El-Masri M, Broyles JB, et al. Testing for viral penetration of non-latex surgical and examination gloves: a comparison of three methods. Clin Microbiol Infect. 2004; 10(4): 3226. 
9. Pratt RJ, Pellowe C, Loveday HP, et al. The epic project: developing national evidence-based guidelines for preventing healthcare associated infections. Phase I: Guidelines for preventing hospital-acquired infections. Department of Health (England). J Hosp Infect. 2001; 47 Suppl: S3-82.

10. Korniewicz DM, El-Masri M, Broyles JM, et al. Performance of latex and nonlatex medical examination gloves during simulated use. Am J Infect Control. 2002; 30(2): 133-8.

11. Neal JG, Jackson EM, Suber F, et al. Latex glove penetration by pathogens: a review of the literature. J Long Term Eff Med Implants. 1998; 8(3-4): 233-40.

12. Phalen R and Hee SQ. A moving robotic hand system for whole-glove permeation and penetration: captan and nitrile gloves. J Occup Environ Hyg. 2008; 5(4): 258-70.
13. Oztan MD, Pekiner BD and Can A. Permeability of latex gloves after exposure to 6 chemical agents. Quintessence Int. 2007; 38(9): e537-43.

14. Gunasekera PC, Fernando RJ and de Silva KK. Glove failure: an occupational hazard of surgeons in a developing country. J R Coll Surg Edinb. 1997; 42(2): 95-7.

15. Tanner $\mathrm{J}$ and Parkinson H. Double gloving to reduce surgical cross-infection. Cochrane Database Syst Rev. 2006(3): CD003087.

16. Ready MA, Schuster GS, Wilson JT, et al. Effects of dental medicaments on examination glove permeability. J Prosthet Dent. 1989; 61(4): 499503. 